\title{
Oral Tecovirimat for the Treatment of Smallpox
}

Douglas W. Grosenbach, Ph.D., Kady Honeychurch, Ph.D., Eric A. Rose, M.D., Jarasvech Chinsangaram, D.V.M., Ph.D., Annie Frimm, B.S., Biswajit Maiti, Ph.D., Candace Lovejoy, B.S., Ingrid Meara, M.S., Paul Long, B.S., and Dennis E. Hruby, Ph.D.

SIGA Technologies, Corvallis, OR

\section{Abstract}

BACKGROUND—Smallpox was declared eradicated in 1980, but variola virus (VARV), which causes smallpox, still exists. There is no known effective treatment for smallpox; therefore, tecovirimat is being developed as an oral smallpox therapy. Because clinical trials in a context of natural disease are not possible, an alternative developmental path to evaluate efficacy and safety was needed.

METHODS-We investigated the efficacy of tecovirimat in nonhuman primate (monkeypox) and rabbit (rabbitpox) models in accordance with the Food and Drug Administration (FDA) Animal Efficacy Rule, which was interpreted for smallpox therapeutics by an expert advisory committee. We also conducted a placebo-controlled pharmacokinetic and safety trial involving 449 adult volunteers.

RESULTS-The minimum dose of tecovirimat required in order to achieve more than $90 \%$ survival in the monkeypox model was $10 \mathrm{mg}$ per kilogram of body weight for 14 days, and a dose of $40 \mathrm{mg}$ per kilogram for 14 days was similarly efficacious in the rabbitpox model. Although the effective dose per kilogram was higher in rabbits, exposure was lower, with a mean steady-state maximum, minimum, and average (mean) concentration $\left(\mathrm{C}_{\max }, \mathrm{C}_{\min }\right.$, and $\mathrm{C}_{\mathrm{avg}}$, respectively) of 374, 25, and $138 \mathrm{ng}$ per milliliter, respectively, in rabbits and 1444, 169, and $598 \mathrm{ng}$ per milliliter in nonhuman primates, as well as an area under the concentration-time curve over 24 hours $\left(\mathrm{AUC}_{0-24 \mathrm{hr}}\right)$ of $3318 \mathrm{ng} \times$ hours per milliliter in rabbits and 14,352 $\mathrm{ng} \times$ hours per milliliter in nonhuman primates. These findings suggested that the nonhuman primate was the more conservative model for the estimation of the required drug exposure in humans. A dose of $600 \mathrm{mg}$ twice daily for 14 days was selected for testing in humans and provided exposures in excess of those in nonhuman primates (mean steady-state $C_{\max }, C_{\min }$, and $C_{a v g}$ of 2209,690 , and $1270 \mathrm{ng}$ per milliliter and $\mathrm{AUC}_{0-24 \mathrm{hr}}$ of 30,632 $\mathrm{ng} \times$ hours per milliliter). No pattern of troubling adverse events was observed.

CONCLUSIONS-On the basis of its efficacy in two animal models and pharmacokinetic and safety data in humans, tecovirimat is being advanced as a therapy for smallpox in accordance with the FDA Animal Rule. (Funded by the National Institutes of Health and the Biomedical Advanced Research and Development Authority; ClinicalTrials.gov number, NCT02474589.)

Address reprint requests to Dr. Hruby at SIGA Technologies, Suite 110, 4575 SW Research Way, Corvallis, OR 97333, or at dhruby@siga.com.

Disclosure forms provided by the authors are available with the full text of this article at NEJM.org. 
Smallpox is caused by variola virus (VARV) ${ }^{1}$ and is highly infectious by the respiratory route. Susceptible persons have up to a $90 \%$ chance of contracting the disease if they are exposed to someone who is infected, ${ }^{2}$ and the overall fatality rate is estimated at $30 \%$ among those who have not been vaccinated. Although the naturally occurring disease was declared eradicated in $1980,{ }^{3}$ smallpox remains a concern because of the potential for deliberate release of VARV as an act of biowarfare or bioterrorism. ${ }^{4}$ The possible existence of clandestine stores of VARV ${ }^{5}$ and the ability to recreate the virus from published genomic sequences through synthetic biologic methods are also of concern. ${ }^{6}$ A single case of smallpox anywhere in the world would be a global health emergency. The available vaccines are not routinely used because of the risk of adverse events, and they would not be used in the general population unless VARV exposure were known or suspected. An antiviral drug against VARV would be beneficial in scenarios in which the vaccine is not effective, such as postexposure prophylaxis 4 to 5 or more days after exposure, before the onset of clinical signs, but most importantly as postexposure therapy after the development of fever and rash.

Because it is not possible to conduct clinical trials involving patients with naturally occurring smallpox and it would be unethical to expose humans to VARV intentionally, the development of tecovirimat has been conducted in accordance with the Food and Drug Administration (FDA) Animal Efficacy Rule, or Animal Rule. The Animal Rule was enacted ${ }^{7}$ to provide an alternative drug-development pathway for diseases in which natural occurrence is rare or nonexistent and ethical concerns do not allow the conduct of more traditional clinical efficacy trials. The rule gives the FDA authority to approve drugs on the basis of animal efficacy data, provided that all other aspects of drug development are completed satisfactorily.

A number of drugs and biologic agents developed under the Animal Rule have been approved by the FDA, although most had previous approvals for related indications. ${ }^{8}$ In December 2012, the FDA approved the monoclonal antibody raxibacumab for treatment of inhalation anthrax as the first new antiinfective drug (biologic) developed solely according to the rule. Since then, the FDA has approved additional biologic agents for prophylaxis or treatment of anthrax (obiltoxaximab [Anthim] and anthrax immune globulin [Anthrasil]) and botulism (botulism antitoxin heptavalent [BAT]). ${ }^{8}$ For each of these products, efficacy was shown in more than one animal model that was accepted by the FDA as reasonably predictive of human response. The exposure-response relationship was determined, and critical pharmacokinetic measures and exposure levels that were correlated with efficacy and target exposures in humans were identified. Drug safety was then assessed in humans at a dose and exposure that were equal to or greater than those found to provide a survival benefit in animals. Tecovirimat development has followed a similar path (Fig. S1 in the Supplementary Appendix, available with the full text of this article at NEJM.org).

\section{METHODS STUDY AGENT}

Tecovirimat (previously ST-246) was identified through screening of a chemically diverse library of more than 350,000 unique compounds ${ }^{9}$ (Fig. S2 in the Supplementary Appendix). The compound specifically inhibits all orthopoxviruses tested in vitro, including VARV 
(mean half maximal effective concentration [EC $\left.{ }_{50}\right], 0.010$ to $0.068 \mu$ mol per liter), with no activity against other viruses. ${ }^{9}$ Tecovirimat inhibits $\mathrm{p} 37$, a protein that is present and highly conserved (approximately 98\% amino acid identity) in all orthopoxviruses; the inhibition of p37 prevents the formation and egress of enveloped virions, which are essential for virulence. ${ }^{10-15}$ For studies in animals, tecovirimat was formulated in a suspension vehicle consisting of $1 \%$ weight per volume hydroxypropyl methylcellulose (HPMC) and $0.5 \%$ weight per volume Tween 80 ready to be administered to the animals by oral gavage. For clinical studies, tecovirimat drug product was supplied for oral administration as immediaterelease opaque hard gelatin capsules, size " 0 ," containing $200 \mathrm{mg}$ of tecovirimat plus excipients. All the inactive ingredients are conventional, United States PharmacopeiaNational Formulary grade, and generally accepted as safe. Additional metabolic and toxicologic findings are described in the Supplementary Appendix.

\section{EFFICACY STUDIES IN ANIMALS}

In pilot studies, tecovirimat was shown to provide protective efficacy in multiple smallanimal and nonhuman primate models of orthopoxvirus disease. ${ }^{9,16-23}$ Subsequently, the efficacy of tecovirimat has been evaluated in lethal intravenous-injection monkeypox and intradermal-injection rabbitpox models of human smallpox, which have been well characterized for the evaluation of smallpox countermeasures ${ }^{24,25}$ (an overview of the animal models and comparisons with human smallpox is provided in the Supplementary Appendix).

Four pivotal studies in nonhuman primates and two pivotal studies in rabbits were conducted. Investigations included a dose exploration to determine the minimum fully effective dose and an infected-animal pharmacokinetic study in both animal models, as well as studies of treatment delay and treatment duration in nonhuman primates. Animal care and use protocols were approved by the institutional animal care and use committees of the institutions at which the studies were conducted (the Southern Research Institute, the Army Medical Institute of Infectious Diseases, and the Lovelace Respiratory Research Institute).

In all studies in nonhuman primates, crabeating macaques were infected on day 0 with a lethal dose of monkeypox virus (Zaire 1979 strain) by intravenous inoculation with $5 \times 10^{7}$ plaqueforming units (PFU) per animal. In the studies in rabbits, New Zealand white rabbits were infected on day 0 with a lethal dose of rabbitpox virus Utrecht by intradermal inoculation with $1000 \mathrm{PFU}$ per animal. Additional methodologic details and overviews of the study design for the studies in nonhuman primates and rabbits are provided in the Animal Study Methods section and Tables S1 through S6 in the Supplementary Appendix. In both models, tecovirimat treatment was initiated on or after day 4 after exposure, after the onset of clinical signs (pock lesions in nonhuman primates and fever and viremia in rabbits). In all studies, survival was the primary end point, and lesion formation (nonhuman primates) and viral load (both animal models) were secondary pharmacodynamic end points.

In the first study in nonhuman primates, tecovirimat doses of 0 (placebo), $0.3,1,3$, and 10 $\mathrm{mg}$ per kilogram of body weight were evaluated for efficacy. The second study examined the pharmacokinetics of tecovirimat in infected nonhuman primates to allow the development of a population pharmacokinetic model in addition to providing a larger sample of animals for 
survival analysis. The tecovirimat regimen included 14 daily doses of 0 (placebo), 3, 10, and $20 \mathrm{mg}$ per kilogram. Two additional studies were conducted in nonhuman primates to evaluate the effect of a delay in the initiation of treatment and of treatment duration. The results of the study of delays in the initiation of treatment were previously reported by Berhanu et al. ${ }^{26}$ All studies included a placebo control group.

In the first study in rabbits, different doses of tecovirimat, including 0 (placebo), 20, 40, 80, and $120 \mathrm{mg}$ per kilogram, were evaluated for efficacy. The second study in rabbits was a pharmacokinetic study in infected rabbits, conducted to develop a population pharmacokinetic model and expand the survival analysis population. In this study, tecovirimat dosing was initiated at 4 days after exposure and included doses of 40,80 , and $120 \mathrm{mg}$ per kilogram.

Pharmacokinetic and pharmacodynamic models were developed for both animal models to establish the tecovirimat exposure-survival response relationship. Model-based and noncompartmental approaches were used to contrast the exposures in nonhuman primates and rabbits treated at the maximally effective dose for each model to identify the more conservative animal model (i.e., the model requiring higher tecovirimat exposures for maximal benefit).

\section{EXPANDED SAFETY TRIAL}

Pharmacokinetic and safety data from phase 1 and phase 2 dose-escalation and repeat-dose studies ${ }^{27,28}$ were used to model the dosing in humans. The expanded safety trial was a multicenter, randomized, double-blind, safety trial involving healthy volunteers 18 to 79 years of age. The chosen dose targeted an exposure level that was a multiple of that required for maximal efficacy in nonhuman primates but below the highest level that had been established as being associated with no adverse effects in toxicologic studies in animals. The institutional review board of each participating center approved the protocols. All the volunteers provided written informed consent. Full details of the trial design are provided in the protocol, available at NEJM.org.

A lead-in cohort of 40 participants was randomly assigned in a 4:1 ratio, in either a fed or a fasting state, to receive $600 \mathrm{mg}$ of tecovirimat administered orally twice daily or matching placebo. After a blinded interim analysis of safety and pharmacokinetic data showing that sufficient levels in blood had been achieved, as well as FDA review of the data, the trial was expanded to provide a large enough database for evaluation of product safety by randomly assigning an additional 412 participants at 11 sites to receive tecovirimat or placebo in the fed state only. Participants recorded all adverse events and concomitant medications in a diary and during interviews with clinic staff from the start of the trial period to completion of the trial at the follow-up visit on day 28. Adverse events were also recorded during physical examinations and through laboratory evaluations on scheduled clinic days. A follow-up visit or telephone contact at day 45 for the evaluation of adverse events was conducted only for participants in whom adverse events or serious adverse events were present on the day 28 follow-up visit. 


\section{RESULTS \\ EFFICACY STUDIES IN ANIMALS}

In aggregate, 1 of 20 nonhuman primates that received placebo survived infection with monkeypox virus and no rabbits that received placebo survived rabbitpox virus infection (Figs. 1 and 2, and Tables S7 and S8 in the Supplementary Appendix). Additional results for viral load and lesion counts are provided in Figures S4, S5, and S6 in the Supplementary Appendix. On the basis of the results of the first two studies in nonhuman primates, the minimum effective dose was determined to be 3 to $10 \mathrm{mg}$ per kilogram (Fig. S3 in the Supplementary Appendix), which provided nearly full protection from death (i.e., a survival rate of approximately 95\%, as compared with 5\% in the placebo group) and reduced viral loads and lesion counts. Subsequent studies were conducted with the dose of $10 \mathrm{mg}$ per kilogram, because this dose reduced viral load and lesion counts to a greater extent than the dose of $3 \mathrm{mg}$ per kilogram.

As previously reported by Berhanu et al., ${ }^{26}$ in the treatment-delay study, a dose of $10 \mathrm{mg}$ per kilogram initiated at 4 or 5 days after exposure resulted in a survival rate of $83 \%$, whereas treatment initiation 6 days after exposure resulted in a survival rate of $50 \%$. In the treatmentduration study, 3 daily doses at $10 \mathrm{mg}$ per kilogram initiated 4 days after exposure resulted in a survival rate of $50 \%$, whereas 5 and 7 daily treatments resulted in a survival rate of $100 \%$. Ten daily treatments resulted in $80 \%$ survival. The pharmacokinetic study in nonhuman primates showed that after the 14th dose (steady state) of $10 \mathrm{mg}$ per kilogram, the mean values in plasma that were associated with efficacy were a maximum concentration $\left(\mathrm{C}_{\max }\right)$ of $1444 \mathrm{ng}$ per milliliter, a minimum concentration $\left(\mathrm{C}_{\min }\right)$ of $169 \mathrm{ng}$ per milliliter, an average (mean) concentration $\left(\mathrm{C}_{\mathrm{avg}}\right)$ of $598 \mathrm{ng}$ per milliliter, and an area under the concentration-time curve over 24 hours $\left(\mathrm{AUC}_{0-24 \mathrm{hr}}\right)$ of 14,352 $\mathrm{ng} \times$ hours per milliliter (Table 1).

In rabbits, the minimum effective dose was determined to be 20 to $40 \mathrm{mg}$ per kilogram, which provided protection from death and illness equivalent to that of the higher doses we evaluated (80 and $120 \mathrm{mg}$ per kilogram) (Figs. 1 and 2, and Fig. S3 in the Supplementary Appendix). The dose of $40 \mathrm{mg}$ per kilogram was selected as the standard for fully effective dosing. A population pharmacokinetic model was developed on the basis of plasma exposures after days 1, 7, and 14 of treatment. The mean values in plasma at steady state that were associated with efficacy after treatment with the dose of $40 \mathrm{mg}$ per kilogram were a $\mathrm{C}_{\max }$ of $374 \mathrm{ng}$ per milliliter, a $\mathrm{C}_{\min }$ of $25 \mathrm{ng}$ per milliliter, a $\mathrm{C}_{\mathrm{avg}}$ of $138 \mathrm{ng}$ per milliliter, and an $\mathrm{AUC}_{0-24 \mathrm{hr}}$ of $3318 \mathrm{ng} \times$ hours per milliliter (Table 1).

Although the efficacious exposure values in nonhuman primates treated with $10 \mathrm{mg}$ per kilogram and in rabbits treated with $40 \mathrm{mg}$ per kilogram were similar after the first dose (ratios of exposure in nonhuman primates as compared with that in rabbits ranged from 1.1 to 1.4), the exposure values in nonhuman primates were higher at steady state, with ratios (nonhuman primates:rabbits) of 3.9 for $\mathrm{C}_{\max }, 6.9$ for $\mathrm{C}_{\min }, 4.3$ for $\mathrm{C}_{\mathrm{avg}}$, and 4.3 for $\mathrm{AUC}_{0-24 \mathrm{hr}}$ (Table 1). These data suggest that the nonhuman primate is the more conservative animal model, since it requires higher exposure for maximum efficacy and would result in a higher predicted dose in humans for achieving efficacy against smallpox. 


\section{TECOVIRIMAT CLINICAL TRIAL}

In the expanded safety trial, 851 volunteers were screened, of whom 452 underwent randomization and were assigned to receive tecovirimat at a dose of $600 \mathrm{mg}$ twice daily (361 participants) or matching placebo (91 participants) for 14 consecutive days (Fig. S7 in the Supplementary Appendix). On the basis of the exposure-response relationship in animal models and previous clinical pharmacokinetic data over a range of doses, ${ }^{27,28}$ modeling and simulation studies ${ }^{29}$ predicted that a dose of $600 \mathrm{mg}$ twice daily would provide exposure in excess of that provided by the efficacious doses in animals. Although studies in animals have shown that five daily doses is the minimum number that is sufficient to provide a survival advantage similar to that associated with longer dosing durations, the 14-day regimen was selected on the basis of the kinetics of the humoral immune response in smallpox, ${ }^{3}$ which is necessary to clear the virus and prevent recrudescence of disease. The demographic and baseline characteristics of the trial participants were well balanced in the trial groups (Table 2). A total of 431 participants completed the trial. The overall rate of adherence was $94.4 \%$ in the placebo group and $93.6 \%$ in the tecovirimat group; the corresponding rates among participants in the pharmacokinetic portion of the trial were $100 \%$ and $96.9 \%$.

The pharmacokinetic profiles and exposures of approximately 48 participants in a fed state are presented to compare with those in the nonhuman primate model (Fig. 1E and $1 \mathrm{~F}$ and Table 1, and Table S9 and Fig. S8 in the Supplementary Appendix). There were 386 nonserious adverse events reported throughout the trial by 164 participants; 208 of these events were thought to be related to tecovirimat or placebo (Table 3, and Table S10 in the Supplementary Appendix). The adverse events related to the trial regimen that occurred or worsened during the trial period were similar in the two groups (Table S11 in the Supplementary Appendix). Adverse events of grade 3 or higher occurred or worsened during treatment at a frequency of $1.1 \%$ in both the tecovirimat group and the placebo group (Table S12 in the Supplementary Appendix) and included headache, osteoarthritis, and hidradenitis. One fatal adverse event was found to be related to a pulmonary embolism that occurred in a participant 1 week after completion of tecovirimat treatment. The participant had a history of recent recurrent deep-vein thromboses but was not being treated with anticoagulant agents. Toxicologic testing revealed no ethanol or drugs of abuse, and the death was judged by the investigators to be unrelated to tecovirimat. Eight participants ( 2 in the placebo group and 6 in the tecovirimat group) discontinued the trial regimen because of adverse events (Table S13 in the Supplementary Appendix).

Pharmacokinetic analysis revealed that the $\mathrm{C}_{\max }$ and $\mathrm{AUC}_{0-24 \mathrm{hr}}$ of tecovirimat under fed conditions were up to $50 \%$ and $45 \%$ greater on day 1 and day 14 , respectively, than they were under fasting conditions (Fig. 1E and $1 \mathrm{~F}$ ). Multiple-day dosing in humans resulted in drug accumulation, with steady-state drug exposures that were approximately $50 \%$ $\left(\mathrm{AUC}_{0-24 \mathrm{hr}}\right)$ and $40 \%\left(\mathrm{C}_{\mathrm{max}}\right)$ higher than those after the first dose. Steadystate levels of tecovirimat were achieved by day 6 , and elimination half-life values were similar (approximately 23 hours) in the fasting and fed groups (Table S9 in the Supplementary Appendix). However, clearance was 55.3 and 39.2 liters per hour in fasting and fed participants, respectively, findings consistent with the previous observation that food altered the presystemic bioavailability of tecovirimat but not the systemic elimination of the drug. ${ }^{30}$ 


\section{DISCUSSION}

Because the risk of VARV infection persists, developing antiviral therapy to treat it is important. Because the smallpox vaccine is no longer used in the general population, herd immunity is minimal, and the vaccine is largely ineffective after the onset of clinical illness. These gaps could be mitigated by an effective smallpox antiviral drug to treat people who have clinically evident disease as well as those who have been exposed or potentially exposed to smallpox. A smallpox antiviral drug could be an important stopgap measure until the standard vaccine can be effectively deployed, while complementing the protective efficacy of the vaccine once it has been deployed.

The eradication of smallpox and the limited usefulness and availability of animal models of VARV infection pose unique challenges in the development and validation of potential smallpox antiviral drugs. In contrast to other Animal Rule investigational scenarios, smallpox antiviral exploration requires the use of surrogate pathogens and hosts for human smallpox. Because of this unique complexity, the FDA convened an advisory panel on the subject of smallpox antiviral-drug development in December 2011. The panel concluded that lethal monkeypox virus infection in nonhuman primates and rabbitpox virus infection in rabbits allowed meaningful evaluation of potential smallpox antiviral drugs, although studies in human volunteers would be necessary to confirm that the doses in humans can safely provide similar exposure to that required for efficacy in animals. The FDA subsequently stipulated that human exposure achieved with drug candidates evaluated under the Animal Rule should provide a multiple of the effective exposure in animals ${ }^{31}$ as an additional threshold to increase the likelihood of efficacy on the basis of animal models.

This report represents the triangulation of safety, pharmacokinetic, and efficacy data from two animal models and noninfected human volunteers (Fig. S1 in the Supplementary Appendix). The efficacy findings in our studies in rabbits confirmed the earlier observations from studies in nonhuman primates that efficacious drug exposures in the nonhuman primate model were higher than required in the rabbit model. Dosing in humans was, therefore, targeted to achieve a multiple of the efficacious exposure in the nonhuman primate model. The exposures achieved with a dose of $600 \mathrm{mg}$ twice daily exceeded the efficacious exposures in nonhuman primates. For $\mathrm{C}_{\min }$ in humans, which has been established as a key pharmacokinetic measure most closely correlated with efficacy in animals, ${ }^{29}$ the exposure was 4 times as high as the maximally efficacious exposure in nonhuman primates. No safety concerns were identified in association with tecovirimat use in human volunteers without smallpox who were receiving a 14-day course. Most reported adverse events were mild, and all events, with the exception of the death, resolved without sequelae. Discontinuation of the trial was rare among the participants. The aggregation of the results from these multiple studies involving animals and humans supports tecovirimat as a potential smallpox antiviral drug.

\section{Supplementary Material}

Refer to Web version on PubMed Central for supplementary material. 


\section{Acknowledgments}

Supported by the Division of Microbiology and Infectious Diseases, National Institute of Allergy and Infectious Diseases, National Institutes of Health (contract HHSN266200600014C), and the Biomedical Advanced Research and Development Authority (contract HHSO100201100001C).

We thank the staff of the Defense Threat Reduction Agency, the National Institutes of Health, the Centers for Disease Control and Prevention, and the Biomedical Advanced Research and Development Authority for their research and development support, input, and advice; the staff of the Food and Drug Administration for open communication and continuing guidance in seeking regulatory approval for tecovirimat; and the many members of the poxvirus research community who provided their expertise, assistance, and encouragement to help advance this program.

\section{References}

1. Virus taxonomy: the classification and nomenclature of viruses - 9th Report of the ICTV. Amsterdam: Elsevier; 2012. (https://talk.ictvonline.org/taxonomy/)

2. Weiss MM, Weiss PD, Mathisen G, Guze P. Rethinking smallpox. Clin Infect Dis. 2004; 39:166873. [PubMed: 15578369]

3. Fenner F, Henderson DA, Arita I, Jezek Z, Ladnyi ID. Smallpox and its eradication. Geneva: World Health Organization; 1988.

4. Henderson DA. The looming threat of bioterrorism. Science. 1999; 283:1279-82. [PubMed: 10037590]

5. Gellman B. Washington Post. Nov 5, 20024 Nations thought to possess smallpox.

6. Kupferschmidt K. Labmade smallpox is possible, study shows. Science. 2017; 357:115-6. [PubMed: 28706017]

7. Food and Drug Administration. New drug and biological drug products: evidence needed to demonstrate effectiveness of new drugs when human efficacy studies are not ethical or feasible. Fed Regist. 2002; 67(105):37988-98. [PubMed: 12049094]

8. CDER drug and biologic animal rule approvals. Mar 30, 2018. (https://www.fda.gov/downloads/ Drugs/DevelopmentApprovalProcess/HowDrugsareDevelopedandApproved/ DrugandBiologicApprovalReports/NDAandBLAApprovalReports/UCM578137.pdf)

9. Yang G, Pevear DC, Davies MH, et al. An orally bioavailable antipoxvirus compound (ST-246) inhibits extracellular virus formation and protects mice from lethal orthopoxvirus challenge. J Virol. 2005; 79:13139-49. [PubMed: 16189015]

10. Blasco R, Moss B. Extracellular vaccinia virus formation and cell-to-cell virus transmission are prevented by deletion of the gene encoding the 37,000-Dalton outer envelope protein. J Virol. 1991; 65:5910-20. [PubMed: 1920620]

11. Gurt I, Abdalrhman I, Katz E. Pathogenicity and immunogenicity in mice of vaccinia viruses mutated in the viral envelope proteins A33R and B5R. Antiviral Res. 2006; 69:158-64. [PubMed: 16406098]

12. McIntosh AA, Smith GL. Vaccinia virus glycoprotein A34R is required for infectivity of extracellular enveloped virus. J Virol. 1996; 70:272-81. [PubMed: 8523536]

13. Payne LG. Significance of extracellular enveloped virus in the in vitro and in vivo dissemination of vaccinia. J Gen Virol. 1980; 50:89-100. [PubMed: 7441216]

14. Smith GL, Vanderplasschen A, Law M. The formation and function of extracellular enveloped vaccinia virus. J Gen Virol. 2002; 83:2915-31. [PubMed: 12466468]

15. Wolffe EJ, Isaacs SN, Moss B. Deletion of the vaccinia virus B5R gene encoding a 42-kilodalton membrane glycoprotein inhibits extracellular virus envelope formation and dissemination. J Virol. 1993; 67:4732-41. [PubMed: 8331727]

16. Duraffour S, Snoeck R, de Vos R, et al. Activity of the anti-orthopoxvirus compound ST-246 against vaccinia, cowpox and camelpox viruses in cell monolayers and organotypic raft cultures. Antivir Ther. 2007; 12:1205-16. [PubMed: 18240860] 
17. Nalca A, Hatkin JM, Garza NL, et al. Evaluation of orally delivered ST-246 as postexposure prophylactic and antiviral therapeutic in an aerosolized rabbitpox rabbit model. Antiviral Res. 2008; 79:121-7. [PubMed: 18479761]

18. Quenelle DC, Buller RM, Parker S, et al. Efficacy of delayed treatment with ST-246 given orally against systemic orthopoxvirus infections in mice. Antimicrob Agents Chemother. 2007; 51:68995. [PubMed: 17116683]

19. Quenelle DC, Prichard MN, Keith KA, et al. Synergistic efficacy of the combination of ST-246 with CMX001 against orthopoxviruses. Antimicrob Agents Chemother. 2007; 51:4118-24. [PubMed: 17724153]

20. Sbrana E, Jordan R, Hruby DE, et al. Efficacy of the antipoxvirus compound ST-246 for treatment of severe orthopoxvirus infection. Am J Trop Med Hyg. 2007; 76:768-73. [PubMed: 17426185]

21. Huggins J, Goff A, Hensley L, et al. Nonhuman primates are protected from smallpox virus or monkeypox virus challenges by the antiviral drug ST-246. Antimicrob Agents Chemother. 2009; 53:2620-5. [PubMed: 19349521]

22. Jordan R, Goff A, Frimm A, et al. ST-246 antiviral efficacy in a nonhuman primate monkeypox model: determination of the minimal effective dose and human dose justification. Antimicrob Agents Chemother. 2009; 53:1817-22. [PubMed: 19223621]

23. Mucker EM, Goff AJ, Shamblin JD, et al. Efficacy of tecovirimat (ST-246) in nonhuman primates infected with variola virus (smallpox). Antimicrob Agents Chemother. 2013; 57:6246-53. [PubMed: 24100494]

24. Adams MM, Rice AD, Moyer RW. Rabbitpox virus and vaccinia virus infection of rabbits as a model for human smallpox. J Virol. 2007; 81:11084-95. [PubMed: 17686856]

25. Earl PL, Americo JL, Wyatt LS, et al. Immunogenicity of a highly attenuated MVA smallpox vaccine and protection against monkeypox. Nature. 2004; 428:182-5. [PubMed: 15014500]

26. Berhanu A, Prigge JT, Silvera PM, Honeychurch KM, Hruby DE, Grosenbach DW. Treatment with the smallpox antiviral tecovirimat (ST-246) alone or in combination with ACAM2000 vaccination is effective as a postsymptomatic therapy for monkeypox virus infection. Antimicrob Agents Chemother. 2015; 59:4296-300. [PubMed: 25896687]

27. Chinsangaram J, Honeychurch KM, Tyavanagimatt SR, et al. Safety and pharmacokinetics of the anti-orthopoxvirus compound ST-246 following a single daily oral dose for 14 days in human volunteers. Antimicrob Agents Chemother. 2012; 56:4900-5. [PubMed: 22777041]

28. Jordan R, Chinsangaram J, Bolken TC, et al. Safety and pharmacokinetics of the antiorthopoxvirus compound ST-246 following repeat oral dosing in healthy adult subjects. Antimicrob Agents Chemother. 2010; 54:2560-6. [PubMed: 20385870]

29. Leeds JM, Fenneteau F, Gosselin NH, et al. Pharmacokinetic and pharmacodynamic modeling to determine the dose of ST-246 to protect against smallpox in humans. Antimicrob Agents Chemother. 2013; 57:1136-43. [PubMed: 23254433]

30. Jordan R, Tien D, Bolken TC, et al. Single-dose safety and pharmacokinetics of ST-246, a novel orthopoxvirus egress inhibitor. Antimicrob Agents Chemother. 2008; 52:1721-7. [PubMed: 18316519]

31. Guidance for Industry. Silver Spring, MD: Center for Biologics Evaluation and Research; Oct, 2015 Product development under the animal rule. 


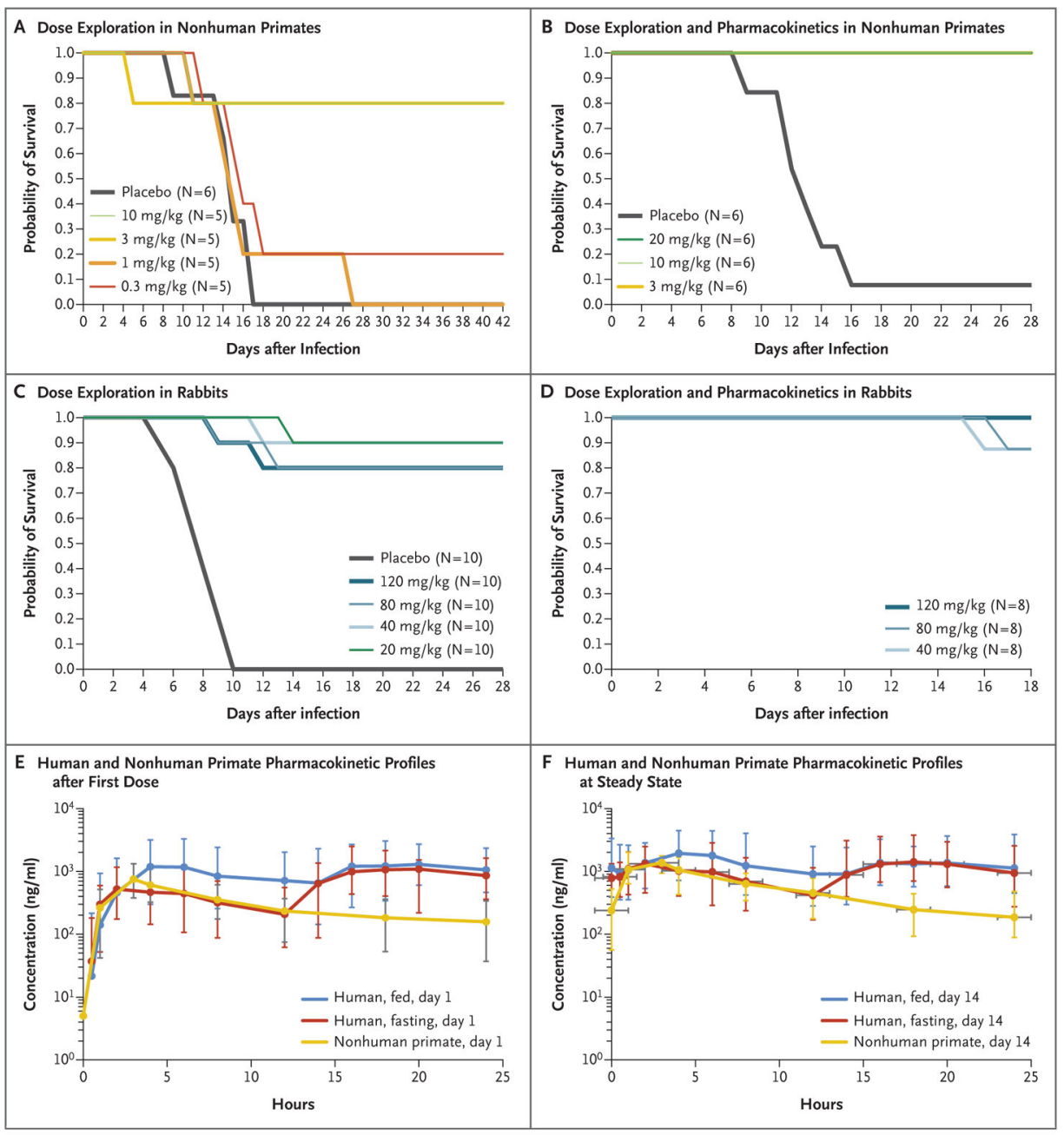

Figure 1. Efficacy and Pharmacokinetic Profiles of Tecovirimat in Animal Models

On day 0, nonhuman primates (Panels A and B) and rabbits (Panels C and D) were infected with a lethal dose of monkeypox virus or rabbitpox virus, respectively. Survival was monitored for 18 to 42 days after infection, indicated on the horizontal axis labels. Tecovirimat was administered by oral gavage at doses ranging from 0.3 to $20 \mathrm{mg}$ per kilogram of body weight in nonhuman primates and from 20 to $120 \mathrm{mg}$ per kilogram in rabbits. Tecovirimat was administered for 14 consecutive daily doses starting on day 4 after infection, after the onset of clinical signs (pock lesions in nonhuman primates and fever and viremia in rabbits) in each study. Comparison of the exposures required for efficacy in rabbits and nonhuman primates showed that the nonhuman primate was the more conservative model, with higher exposures required for full effectiveness. Therefore, the exposure profiles of tecovirimat in plasma in nonhuman primates and humans were compared after the first dose (Panel E) and after the last dose at steady state (Panel F) to evaluate whether exposures in humans exceeded those in nonhuman primates, providing a reasonable expectation of efficacy in humans. 


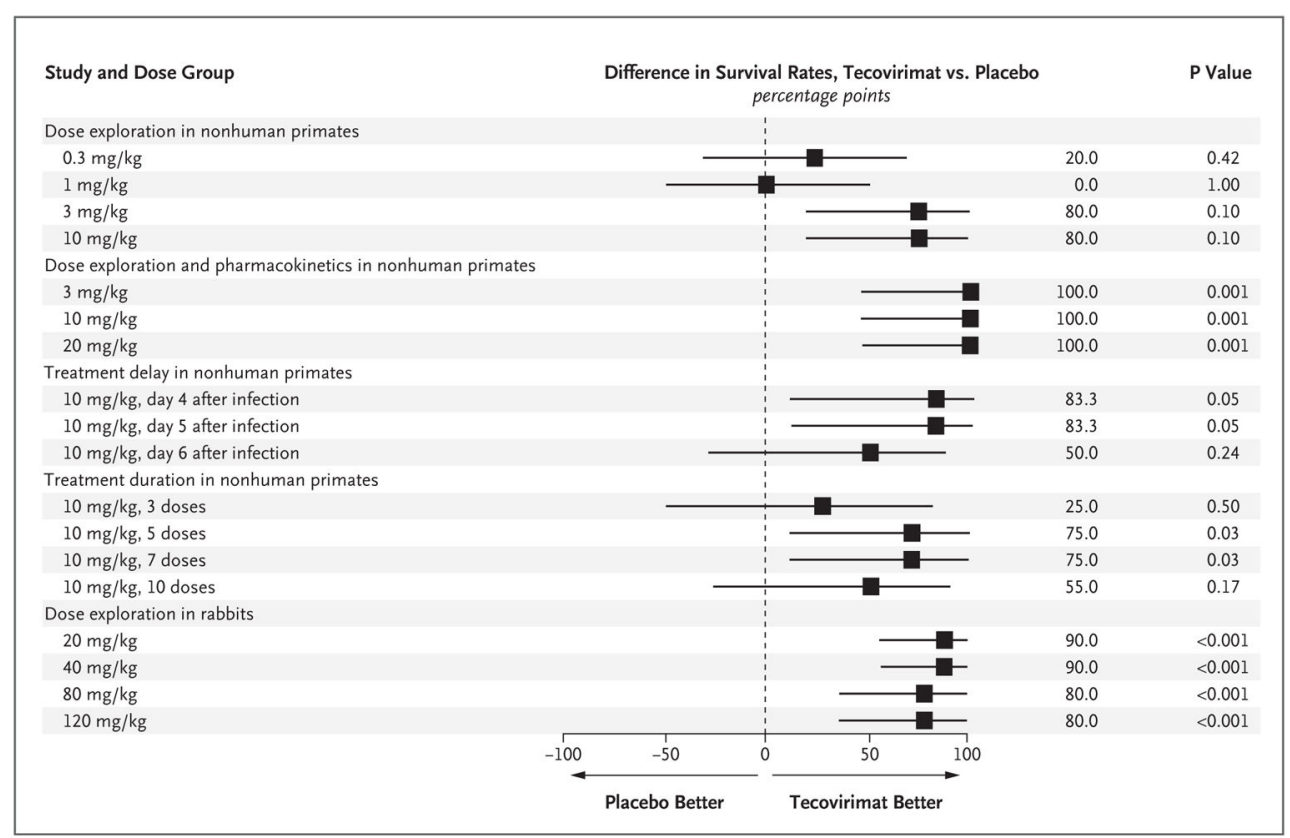

Figure 2. Differences in Survival Rates with Tecovirimat as Compared with Placebo

Shown is a forest-plot summary comparing differences in survival rates between tecovirimat and placebo in each study. The exact $95 \%$ confidence intervals (horizontal bars in the forest plot) are based on the score statistic of the difference in survival rates. The $\mathrm{P}$ value is from a one-sided Fisher's exact test for the comparison of tecovirimat with placebo. Data from the study of dose exploration and pharmacokinetics in rabbits are not shown on the forest plot, since the study did not include a placebo control. 


\section{Table 1}

Geometric Mean of Noncompartmental Exposures in Nonhuman Primates, Rabbits, and Humans Identification of the Most Conservative Animal Model and Comparison with Human Exposures. *

\begin{tabular}{|c|c|c|c|c|c|}
\hline Comparison & $\mathrm{C}_{\max }$ & $\begin{array}{c}\mathrm{C}_{\min } \\
n g / m l\end{array}$ & $\mathrm{C}_{\mathrm{avg}}$ & $\begin{array}{l}\mathrm{AUC}_{0-24 h r} \\
n g \times h r / m l\end{array}$ & \\
\hline \multicolumn{5}{|l|}{ Efficacious exposure in animal models } & \\
\hline \multicolumn{6}{|l|}{ Day 1 (first dose) } \\
\hline Nonhuman primate, $10 \mathrm{mg} / \mathrm{kg}$ daily & 749 & 158 & 318 & 7,629 & \\
\hline Rabbit, $40 \mathrm{mg} / \mathrm{kg}$ daily & 518 & 144 & 292 & 7,004 & \\
\hline Nonhuman primate:rabbit ratio & 1.4 & 1.1 & 1.1 & 1.1 & \\
\hline \multicolumn{6}{|l|}{ Day 14 (steady state) } \\
\hline Nonhuman primate, $10 \mathrm{mg} / \mathrm{kg}$ daily & 1444 & 169 & 598 & 14,352 & \\
\hline Rabbit, $40 \mathrm{mg} / \mathrm{kg}$ daily & 374 & 25 & 138 & 3,318 & \\
\hline Nonhuman primate:rabbit ratio & 3.9 & 6.8 & 4.3 & 4.3 & \\
\hline \multicolumn{5}{|l|}{ Nonhuman primate and human exposure ${ }^{\dagger}$} & \\
\hline \multicolumn{6}{|l|}{ Day 1 (first dose) } \\
\hline Human, $600 \mathrm{mg}$ twice a day & 1591 & 560 & 924 & 25,876 & \\
\hline Nonhuman primate, $10 \mathrm{mg} / \mathrm{kg}$ daily & 749 & 158 & 318 & 7,629 & \\
\hline Human:nonhuman primate ratio & 2.1 & 3.5 & 2.9 & 3.4 & \\
\hline \multicolumn{6}{|l|}{ Day 14 (steady state) } \\
\hline Human, $600 \mathrm{mg}$ twice a day & 2209 & 690 & 1270 & 30,632 & \\
\hline Nonhuman primate, $10 \mathrm{mg} / \mathrm{kg}$ daily & 1444 & 169 & 598 & 14,352 & \\
\hline Human:nonhuman primate ratio & 1.5 & 4.1 & 2.1 & 2.1 & \\
\hline
\end{tabular}


Table 2

Demographic and Clinical Characteristics of the Participants at Baseline (Safety Population).*

\begin{tabular}{|c|c|c|c|}
\hline Population and Characteristic & Placebo $(\mathbf{N}=90)$ & Tecovirimat $(\mathrm{N}=359)$ & Total $(N=449)$ \\
\hline \multicolumn{4}{|l|}{ Sex - no. $(\%)$} \\
\hline Male & $36(40)$ & $148(41)$ & $184(41)$ \\
\hline Female & $54(60)$ & $211(59)$ & $265(59)$ \\
\hline \multicolumn{4}{|l|}{ Race - no. $(\%)^{\dagger}$} \\
\hline White & $62(69)$ & $249(69)$ & $311(69)$ \\
\hline Black & $26(29)$ & $101(28)$ & $127(28)$ \\
\hline Asian & $1(1)$ & $3(1)$ & $4(1)$ \\
\hline American Indian or Alaska Native & 0 & $3(1)$ & $3(1)$ \\
\hline Native Hawaiian or other Pacific Islander & $1(1)$ & $1(<1)$ & $2(<1)$ \\
\hline Other & 0 & $2(1)$ & $2(<1)$ \\
\hline \multicolumn{4}{|l|}{ Ethnic group - no. $(\%)^{\dagger}$} \\
\hline Hispanic or Latino & $5(6)$ & $43(12)$ & $48(11)$ \\
\hline Not Hispanic or Latino & $85(94)$ & $315(88)$ & $400(89)$ \\
\hline Other & 0 & $1(<1)$ & $1(<1)$ \\
\hline \multicolumn{4}{|l|}{ Age $-\mathrm{yr}^{t^{t}}$} \\
\hline Mean & $41.9 \pm 15.8$ & $40.4 \pm 15.7$ & $40.7 \pm 15.7$ \\
\hline Median & 40.5 & 38.0 & 39.0 \\
\hline Range & $18-80$ & $18-79$ & $18-80$ \\
\hline \multicolumn{4}{|l|}{ Weight $-\mathrm{kg} \xi$} \\
\hline Mean & $89.7 \pm 29.2$ & $88.6 \pm 22.8$ & $88.8 \pm 24.2$ \\
\hline Median & 84.0 & 85.4 & 85.1 \\
\hline Range & $51.4-240.6$ & $49.1-188.0$ & $49.1-240.6$ \\
\hline \multicolumn{4}{|l|}{ Height $-\mathrm{cm} \delta \mathscr{I}$} \\
\hline Mean & $168.7 \pm 9.0$ & $169.3 \pm 9.4$ & $169.2 \pm 9.3$ \\
\hline Median & 168.2 & 168.7 & 168.7 \\
\hline Range & $152.4-190.5$ & $144.7-194.3$ & $144.7-194.3$ \\
\hline \multicolumn{4}{|l|}{ Body-mass index $s \mathscr{I} / /$} \\
\hline Mean & $31.5 \pm 9.7$ & $30.9 \pm 7.6$ & $31.0 \pm 8.1$ \\
\hline Median & 29.0 & 29.4 & 29.3 \\
\hline Range & $19.5-78.1$ & $17.4-61.1$ & $17.4-78.1$ \\
\hline
\end{tabular}

Plus-minus values are means $\pm \mathrm{SD}$.

${ }^{\dagger}$ The categories for race and ethnic group were the standard categories used by INC Research (now Syneos Health), the company that conducted the clinical trial. Participants chose their answers from a drop-down menu; if the category "Other" was chosen, the participant was asked to specify the answer.

${ }^{7}$ Age was calculated as the date of the day 1 visit minus the date of birth, divided by 365.25 . 
$\xi_{\text {Measurement was performed at screening. }}$

II Data were missing for one participant in the tecovirimat group.

/Body-mass index is the weight in kilograms divided by the square of the height in meters. 


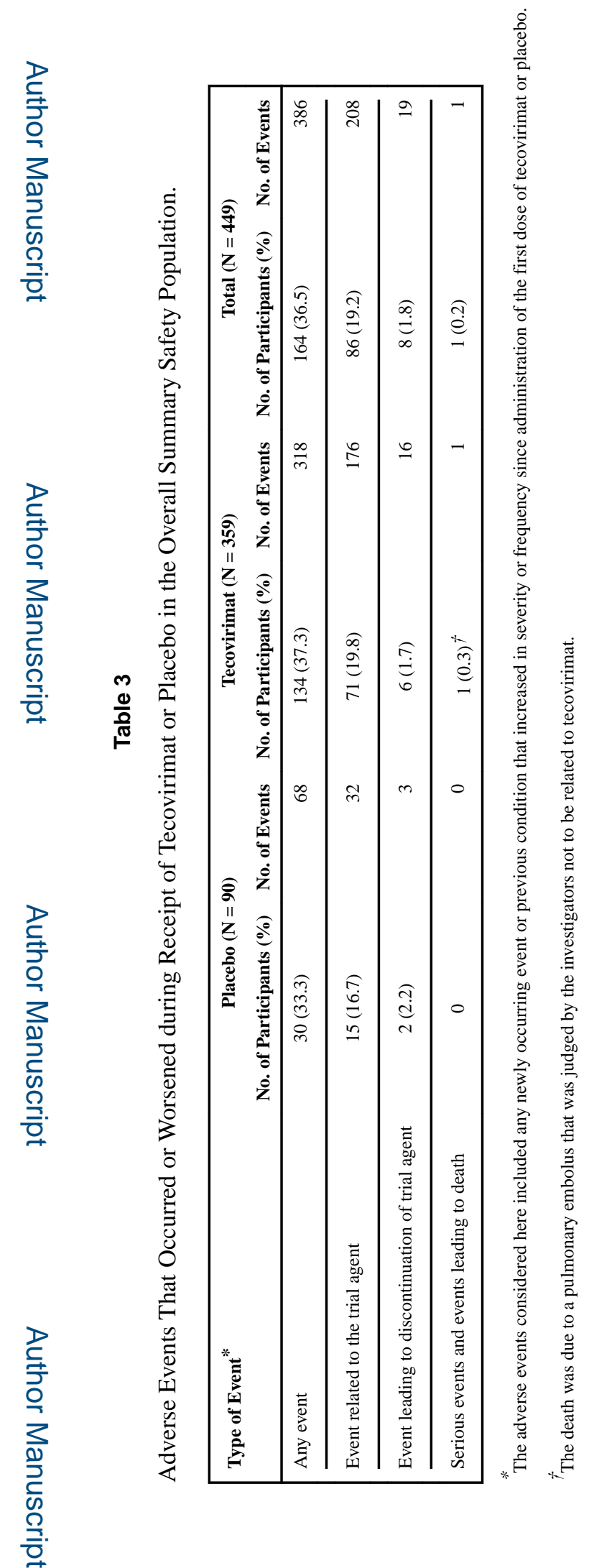

N Engl J Med. Author manuscript; available in PMC 2019 January 05. 